\title{
Effect of Robot and Screen Agent Recommendations on Human Decision-Making
}

\author{
Kazuhiko Shinozawa ${ }^{1}$ and Junji Yamato ${ }^{2}$ \\ ${ }^{1}$ ATR Intelligent Robotics and Communication Laboratories, \\ ${ }^{2}$ NTT Communication Science Laboratories \\ Japan
}

\section{Introduction}

Two-dimensional (2D) character agents that have a human-like appearance are being developed. In the future, such agents will be able to interact with their users in a natural and friendly manner through speech recognition, synthesized speech, and $\square$ action displays. In addition, robots or robotic companions that have a three-dimensional (3D) physical body are attracting attention as communication partners.

Such embodied social agents (ESAs) make interaction more meaningful than it is when interfaces do not appropriately display actions or speak (Beskow and McGlashan, 1997). It is known that people's attitudes towards computerized media are similar to the attitudes they have towards other people (Reeves and Nass, 1996). Even if people only read text or hear a voice from computers, they tend to assign some social existence to them. More social richness, defined as more complete human-like presentations, promises to make computers more attractive, productive, and easy to use. Some research has provided fruitful results and ¿ suggestions for presentation, i.e., graphical appearance (Massaro, 1998), non-verbal behavior (Cassell and Thórisson, 1999), and speech characteristics (Nass and Gong, 1999), and for @ personality (Nass and Isbister, 1998), emotion (Ball and Breese, 1998; Becheiraz and 들 Thalmann, 1998), ethnicity (Takeuchi et al., 1998), and interpersonal communication strategy ¿ (Shinozawa et al., 2001) as well. Much of such research suggests that an ESA should be an Q effective interface for interactions with media.

The above research mainly focused on graphical on-screen agents and computers. On the other hand, robots having a physical body have attracted some attention as useful physical $\$$ agents, and the above research results may apply to interaction with such robots. However, क् when we consider robots as ESAs, a new research topic, “dimensionality", appears. A robot $\stackrel{0}{\widetilde{O}}$ has a $3 \mathrm{D}$ physical body while an on-screen agent has a $2 \mathrm{D}$ one. This leads to several స్ questions: Does increasing dimension make a big difference or not? Does the physical 3D appearance affect us in a significant way during the interaction? When both a 2D agent like Of an on-screen agent and a physical 3D agent like a robot have a similar shape and use the O same voice, what is the significance of the difference in dimensionality? Little research has focused on dimensionality, and we still have no solid answers. We live and work in 3D ญे space. Everything has three dimensions and is located in 3D space. With a 3D body, Oे pointing to some location makes it easy to understand what is being pointed at. When a 
robot navigates a person, the combination of the robot's gestures and its body's direction has a strong relationship with high ratios of successful task completion (Ono et al., 2001). This suggests that the dimensionality produces a difference in the effect of interaction and that a 3D body makes interaction more meaningful.

How can we quantitatively measure the dimensionality or eye movement effects? Almost all research exploring the behavioral factor's effects of ESAs has been conducted by using questionnaire-based evaluations. For example, subjects are asked whether a robot is familiar or not. Getting a feeling of familiarity is important for a pet-like partner. However, it is not enough for a communication partner. Whenever we engage a communication partner, we listen to what the partner has to say, respond to it, and sometime change our thinking. If an ESA's behavior can influence human decision-making, it leads that he/she treats it as a communication partner. This would be one of evaluations for a communication partner. So, we mainly investigate an ESA's influence on human decision-making for evaluating above factors.

In this chapter, in an attempt to answer some the above questions, we discuss dimensionality, investigated by directly comparing results between an on-screen agent and a physical robot, and the role of a robot's eye movement in human-robot interaction. On both topics, the discussions are based on a quantitative evaluation of each factor's effect on human decision-making with a selection task. Section 2 describes the selection task with the direct comparison topic and presents the experimental results. Section 3 describes the effect of a tracking function in the 3D world case and presents the results. Section 4 discusses the effect of the dimensionality and eye movement with the experimental results. Then, Section 5 concludes with a short summary.

\section{Difference in 3D and 2D agent's recommendation}

Recommendation in an advertisement and assistance in a navigation task are two typical situations influencing human decision-making. An ESA acting as an assistant can easily influence users' decisions because users want to know appropriate information. Generally speaking, however, changing a user's mind is difficult in the advertisement situation. Advertising is an important application of ESAs, and we can also say that the recommendation includes helpful interaction-like assistance, because the initial recommendation does not always depend on what the user wants. So, we focus on the advertisement situation and measure the influence of ESAs on human decision-making.

\subsection{Color-name selection task}

The color-name selection task was introduced to quantitatively measure the influence of ESAs on human decision-making in an advertisement situation (Shinozawa et al., 2001). In a color-name selection task, a subject looks at a colored region and selects the color name from two options. The matching ratio of the recommended color names is measured. The ratio is treated as showing the degree of a recommendation's influence on human decision-making.

\subsection{Recommendation situation}

In the case of direct comparison between an on-screen agent and a physical robot, two situations are considered. In one, an ESA points to an object located in 3D space and in the other, it points to an object in 2D space during interaction. Accordingly, we prepared two scenes for interacting with an ESA. 
One scene is equivalent to the original one in the color-name selection task (Shinozawa et al., 2001). An ESA recommends a color name while it points to or looks at the color region and two color-name options on a CRT display when a subject should select one of them. In this case, objects used in the selection task are mainly located in 2D space. We therefore call this, the $2 \mathrm{D}$ world condition. The other scene is a new one that we call the $3 \mathrm{D}$ world condition. The color region that an ESA points to is in 3D space. Actually, we developed two new machines for displaying color regions in 3D space. One displays printed color plates according to external PC control. The other is a button box for displaying and selecting a color name. In the 3D world condition, a subject looks at printed color plates and selects color names using the button box (Fig. 1).

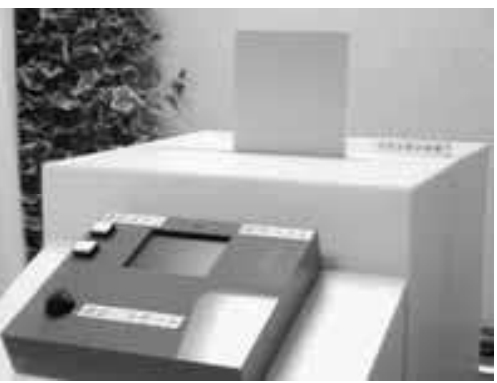

Figure 1. Display machines

The ESA recommended one of the two options under these two conditions, and we investigated the dimensionality by comparing the ESA's effect on user decision-making.

\subsection{Robot and On-screen agent}

Appearance is important for robots as well as for on-screen agents. Humans tend to recognize social roles, gender, or characters by analogy with appearance. Prior knowledge according to appearance has much influence on subjective evaluation (Shibata and Tanie, 2001).

In dimensionality issue, to avoid such influence, we made the appearance of robots and onscreen agents as equivalent to each other as possible [Fig. 2(a) and (b)]. The robot's height is $300 \mathrm{~mm}$ and on-screen agent's height is similar to the robot's one.

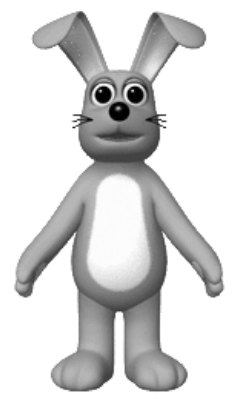

(a) On-screen agent

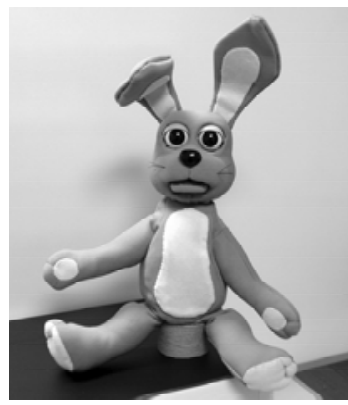

(b) Robot

Figure 2. Appearance of ESAs

Similarly, voice plays an important role in molding the robot's or on-screen agent's character. Both the robot and on-screen agent use the same voice, which was made by 
"Fluet", a Japanese speech synthesizer developed by NTT (Mizuno and Nakajima, 1998). The robot was also developed by NTT.

\subsection{Gestures}

We prepared 27 gestures for both the robot and on-screen agent, which included pointing to a color region, nodding, blinking, and so on. We made the robot's motions similar to the onscreen agent's motions.

\subsection{Color names}

Before starting an experiment, subjects were told that this was a color-name selection task and that they should make a selection based on their own feeling and that there were no correct answers. Most of the color regions and options for color names in the experiment, such as vermilion or carmine, are unfamiliar to ordinary people.

\subsection{Speaking words}

The robot and agent offered their personal opinions, for example, "I think it's vermilion", "This shade is vermilion, isn't it?", instead of making statements that would indicate it had definite knowledge about the displayed color. This was to avoid the effect of the subject's attributing any authority to the robot and agent.

\subsection{Recommended color names}

We carried out pretests without an ESA's recommendation and determined what color names the ESA should recommend and the orders of color name options. In both the 2D and $3 \mathrm{D}$ world condition, the same order of color names and the same recommended color name options were used.

\subsection{Face direction when ESAs speak}

Whenever we talk to someone, we look at that person's face or eyes. ESAs should behave in the same way. With an on-screen 2D agent, a well-known illusion associated with full-faced portraits occurs: from any viewing angle, it appears that the agent's gaze is always on the user (Bruce \& Young, 1998). An on-screen agent's full-face animation can give a feeling that the agent talks to subjects. However, humans can easily detect that the eyes of a 3D face's are not looking at their face even if the difference from the correct direction is small. We therefore developed a subject's head tracking function by which a robot adjusts its head direction so that its head faces subject's head position.

\subsection{Displayed Color region}

In both world cases, the size of the displayed color region was about $270 \mathrm{~mm} \times 160 \mathrm{~mm}$, and the average distance from subjects to the color regions was about $600 \mathrm{~mm}$. In the 2D world case, the colors displayed on the CRT were measured with the CRT color analyzer three times a day. The changes in these values were small (less than 10\%) for the whole experiment. In the 3D world case, the colors displayed on printed plates were measured with a tristimulus colorimeter once a day. The changes in these values were also small (less than $7 \%$ ) for the entire experiment.

Therefore, all of the subjects saw the same color in each world case. 


\subsection{Subjects}

Six experiments were conducted to manage all combinations described above.

1. 2D world condition

(a) No recommendation (Group No2)

(b) Agent recommendation (Group Ag2)

(c) Robot recommendation (Group Ro2)

2. 3D world condition

(a) No recommendation (Group No3)

(b) Agent recommendation (Group Ag3)

(c) Robot recommendation (Group Ro3)

None of the subjects were experts on color names and all were recruited from the general public. Each subject participated in only one experiment; never more than one. Table 1 shows information about the subjects in each group.

\begin{tabular}{|l|l|l|l|l|l|l|}
\cline { 2 - 7 } \multicolumn{1}{c|}{} & \multicolumn{7}{c|}{ Group } \\
\cline { 2 - 7 } \multicolumn{1}{c|}{} & No2 & Ag2 & Ro2 & No3 & Ag3 & Ro3 \\
\hline Number & 30 & 30 & 30 & 31 & 27 & 30 \\
\hline Mean age & 23.87 & 27.60 & 23.30 & 25.40 & 25.00 & 26.29 \\
\hline Max. age & 49 & 39 & 36 & 36 & 39 & 45 \\
\hline Min. age & 19 & 19 & 18 & 18 & 20 & 18 \\
\hline Ratio of males & 0.43 & 0.50 & 0.50 & 0.50 & 0.44 & 0.48 \\
\hline
\end{tabular}

Table 1. Subjects in each group

\subsection{No recommendation case}

To investigate the influence of an ESA's recommendation on user decisions, we must know the mean of the matching ratios without recommendation. We therefore conducted norecommendation experiments for the $2 \mathrm{D}$ and $3 \mathrm{D}$ world conditions. In these experiments, subjects did not see any on-screen agents or robots and chose a color name with no recommendation. In all recommendation conditions, the recommended color name options were fixed due to the pretest described above. The difference in matching ratios between the no-recommendation case and recommendation case shows the degree of the recommendation's influence on user decision-making. When matching ratios in the recommendation case are greater than in the no-recommendation case, the influence is considered to be positive.

\subsection{Procedure}

Upon arriving at the lab, subjects were told that the purpose of this experiment was to mainly investigate the relationship between color regions and color names, and that they should make a selection based on their own feeling because there were no correct answers. After this explanation, they signed an informed consent statement.

\subsubsection{No-recommendation}

In the no-recommendation condition, subjects did the task without the ESA's recommendation and there was no ESA near them. 


\subsubsection{Recommendation case}

They encountered the robots or on-screen agents for the first time when they entered the experimental room. The ESA behaved like it was asleep until the subject pushed a button. Once the button had been pushed, the ESA behaved like it had been awakened and introduced itself, and the experiment started.

The experiment consisted of thirty questions, and each question had two possible responses. When presenting each question, the ESA made a statement endorsing one of the two possible responses. While the ESA was asking the question and presenting the two choices, these options appeared on the computer display in the 2D world condition and on the button box display in the 3D world condition. The subject in both conditions indicated his/her choice by clicking a radio button on the computer display and by pressing the corresponding button. The subject then pressed the "OK" button to send the selection to the computer.

If the choice matched the ESA's suggestion, the ESA nodded with approval while expressing a positive statement. If it did not match the suggestion, the ESA bowed and shook its head slowly while responding with a negative statement. This continued until all questions were answered. When the interaction finished, the experimenter gave the subject a questionnaire.

Figures 3(a) and (b) show scenes of the on-screen agent and robot experiment in the 2D world condition, respectively.

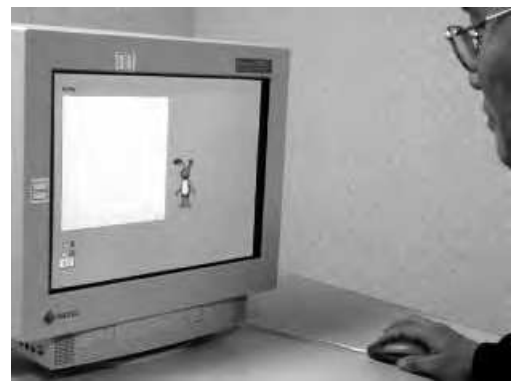

(a) Agent recommendation

Figure 3. Scene in 2D world case

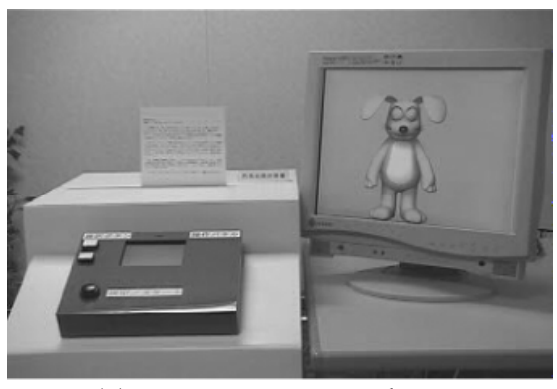

(a) Agent recommendation

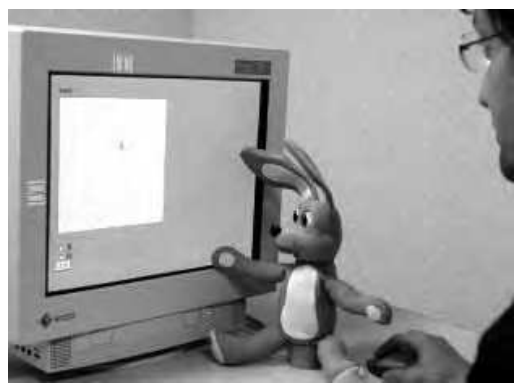

(b) Robot recommendation

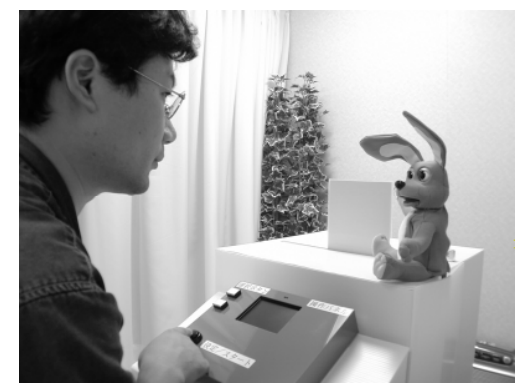

(b) Robot recommendation

Figure 4. Scene in 3D world case

Almost all subjects finished one experiment in less than 20 minutes. The options that subjects selected were automatically recorded in a computer when subjects pushed the OK button. The scenes in one experiment were videotaped. 


\subsection{Results}

We calculated the mean matching ratios of the color names that the agent or robot successfully recommended to each subject. The mean ratios in the groups were also calculated. In the no-recommendation case, subjects did not get any recommendation, but the same color name options as in the recommendation case were presented. In the recommendation case, one of the color names was recommended. In estimating the mean matching ratios in the no-recommendation case, the mean matching ratios of the color names that were recommended in the recommendation case were calculated.

\subsubsection{D world case}

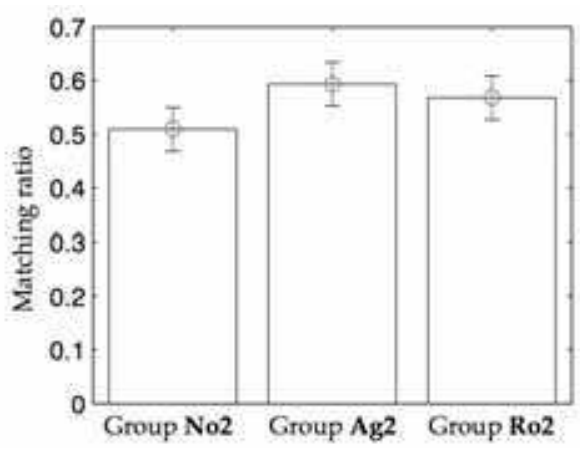

(a) 2D world case

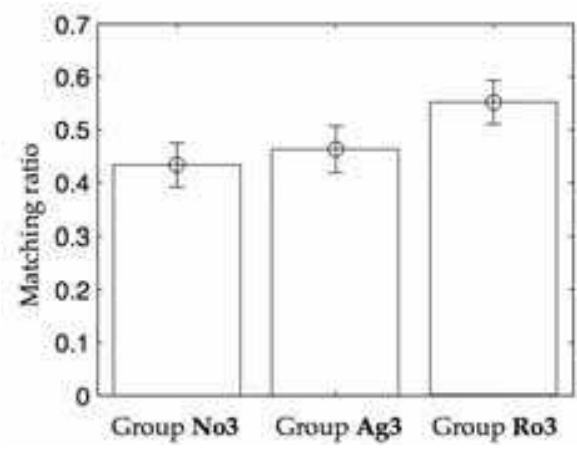

(b) 3D world case

Figure 5. Matching ratios

Figure 5(a) shows the mean matching ratios for each group in the 2D world case. Factorial analyses of variance (ANOVA) were conducted for each mean of matching ratios. We compared the mean between no-recommendation, on-screen agent's recommendation, and robot's recommendation. There was a significant difference only between Group No2 and Group Ag2 (ANOVA, F=3.457, p=0.036, Scheffé, $\mathrm{p}=0.043$ ). The difference between Group No2 and Group Ro2 was not statistically significant.

\subsubsection{D world case}

Figure 5(b) shows the mean matching ratios for each group in the 3D world case. Again, factorial analyses of variance (ANOVA) were conducted for each mean of matching ratios. We again compared the mean between no-recommendation, on-screen agent's recommendation and robot's recommendation. There were significant differences between Group No3 and Group Ro3 and between Group Ag3 and Group Ro3 (ANOVA, F=6.725, $\mathrm{p}=0.002$, Scheffé, $\mathrm{p}=0.003, \mathrm{p}=0.042$ ).

Table 2 summarizes the experiment results. The circles mean that the difference from the no-recommendation case is statistically significant.

\begin{tabular}{|l|c|c|}
\hline & 2D world case & 3D world case \\
\hline Agent & $\bigcirc$ & \\
\hline Robot & - & \\
\hline
\end{tabular}

Table 2. Effect of recommendation 


\section{Gaze effect in 3D agent and 3D world case}

We used the robot with the subject's head tracking function. In the actual experimental situation, subjects sat at fixed place and the relative position from a robot to the place was also fixed. Whenever the robot turned to the place with a fixed angle, its eyes could roughly catch the subject's face or head. However, subjects' sitting posture was not always same and their head sometime slightly moved during the experiment. Even in such a situation, does a robot need a tracking function? Is precisely facing a human head important for a 3D agent? We conducted additional experiments to confirm the importance of having the robot directly facing subjects and investigated whether the importance also holds in a robot with a different appearance. The experiments consisted of three groups with same color-name selection task as in the above experiments. One group used the same rabbit-like robot without a tracking function. The robot could not adjust its head direction to the subject's head movement and always made a same motion. For the other groups, a head robot made a recommendation instead of a rabbit-like robot. Figure 7 shows the head robot's appearance. The robot was developed by MIT AI Laboratory and modified by NTT Communication Science Laboratories. The robot has only a head and neck (no arms or legs), both with 30 degrees of freedom. The robot can produce various facial expressions. In addition, each eyeball has a camera that can pan and tilt. Having cameras inside the eyeballs can makes the robot's gaze direction clear to subjects and ensures the center of the robot's eye can be directed toward subjects with an appropriate vision system. To enable the tracking function, a skin-color region was detected using a detector developed by MIT AI $\mathrm{Lab}$, and the eye direction turned to the center of that region. Table 3 summarizes the three conditions. Subjects' mean age was 24.2 years.
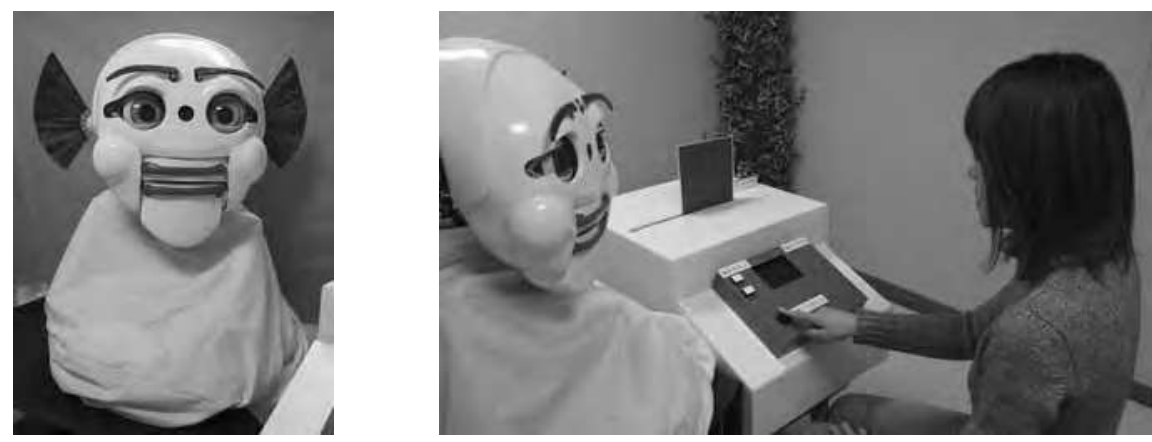

Figure 7. Head robot

\begin{tabular}{|l|l|l|l|}
\cline { 2 - 4 } \multicolumn{1}{c|}{} & \multicolumn{3}{c|}{ Group } \\
\cline { 2 - 4 } \multicolumn{1}{c|}{} & Ro3-2 & Ro3-3 & Ro3-4 \\
\hline Tracking/Non-tracking & Non-Tracking & Non-tracking & Tracking \\
\hline Number & 30 & 14 & 14 \\
\hline Mean age & 20.97 & 25.07 & 23.21 \\
\hline Max. age & 25 & 32 & 35 \\
\hline Min. age & 19 & 19 & 19 \\
\hline Ratio of males & 0.5 & 0.50 & 0.50 \\
\hline
\end{tabular}

Table 3. Groups 


\subsection{Result in matching ratio}

The mean of the matching ratio to the robot's recommended options for each subject is shown in Fig. 8. Factorial ANOVA were conducted for each mean of matching ratios. We compared the mean between non-recommendation, without tracking, and with tracking for the same robot. There is a significant difference in the mean between non-recommendation and the robot with tracking for both robots. (In the rabbit robot case, ANOVA, F $=6.292, \mathrm{p}=$ 0.003 , Scheffé, $p=0.003$ and in the head robot case, ANOVA, $F=4.759, p=0.012$, Scheffé, $p$ $=0.015$ ). The difference between the non-recommendation case and without tracking is not statistically significant $(\mathrm{p}>0.1)$.

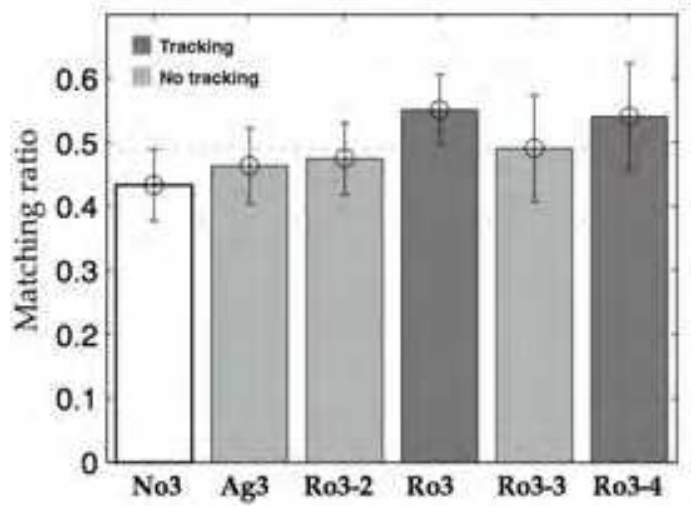

Figure 8. Matching ratios

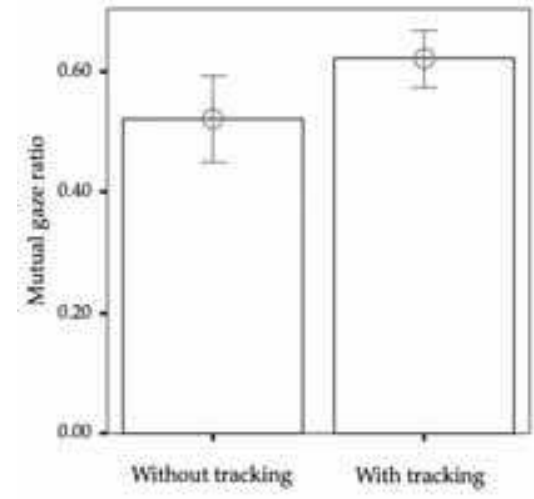

Figure 9. Mutual gaze ratios

\subsection{Result in mutual gaze}

We determined a gaze-period according to the directions of both the robot's gaze and subject's gaze. Subjects' gaze was classified into four categories: "color plate", "button box", "robot's face", and "other". The robot's gaze was classified into three categories: "color plate", "subject's face", and "other". This is because the robot could make its eyes turn toward the button box. In the head robot condition, we recorded the experiment scenes using the cameras equipped in head robot's eyes, which gave us an accurate recording from 
the robot's viewpoint. All assignments were made on the basis of an experimenter's observations with those videos. We assumed that a combination of a robot's gaze to a subject's face and a subject's gaze to a robot's face achieved mutual gaze. We defined the mutual gaze period as the period for which the combination continues. Figure 9 shows the mean ratio of mutual gaze periods for the whole experiment. The ratio in the tracking condition is significantly larger than in non-tracking condition (ANOVA, F=6.468, p=0.018).

\section{Discussion}

Figure 5 shows that the dimensionality of the ESA causes differences in the recommendation's effect on user decision-making. The 3D body was not always superior to the 2D body for recommendation, and on-screen agents seem to have weak points, too.

Those differences cannot be explained only by the advantages or disadvantage of pointing. In the $2 \mathrm{D}$ world condition, the color region was presented on the computer display. The display was in 3D space, so the color region was presented in the 3D space. From this point of view, there should be no difference between the $2 \mathrm{D}$ and $3 \mathrm{D}$ world conditions and therefore no difference in the effect. There must therefore be some other reasons.

The results changed according to the combination of the location pointed to and the ESA's dimensionality. This seems to be evidence supporting the importance of consistency in the dimensionality between communication partners and the environment consisting of the pointing location and manipulated objects. In the 2D world condition, the color region was displayed on the CRT and color name options that should be selected were also on the CRT. In the $2 \mathrm{D}$ world and on-screen agent case, all were contained in the frame of the computer display. The frame might have emphasized the appropriate consistency and the on-screen agent might have had a strong influence through its recommendation. In the 3D world condition, the communication environment was in the 3D physical space. In the 3D world and robot case, the color region and color name options were contained in the physical 3D space, although they were physically separated and there was no frame.

In addition, the effect of robot's recommendation was much greater than that of the onscreen agent's. The robot's body might have had a strong influence for emphasizing appropriate consistency to $3 \mathrm{D}$ space without a visual frame. In addition, behavior in communication contains ambiguous meanings and depends on the situation and communication environment (Sperber and Wilson, 1993). So, humans tend to quickly recognize an environment where the communication partner exists for interpretation.

The results of our experiment seem to provide evidence that humans tend to quickly recognize communication environments even in interaction with an ESA, and also suggest that we must not forget the communication environment in designing ESA behavior.

\subsection{Tracking function effect in 3D world case.}

Figure 8 shows that the influence of a robot without a tracking function is still larger than both 2D agent's and no-recommendation case, although the difference is not significant. The result suggests that the dimensionality still works even if a robot has no tracking function, although the effect is reduced. And, 3D shape with predetermined motions is not sufficient for significantly producing the robot's dimensionality effect on human decision-making. In addition, those results also suggest that only a tracking function is not sufficient for 
explaining all effects caused by the dimensionality, but that a tracking function must be important for robot's influencing human decision-making.

Much research has pointed out eye direction's importance in conversation. For example, our gaze is one way of encouraging someone to talk (Michael \& Mark, 1976). Gaze fixation exerts a special pressure to communication. In addition, research based on questionnaires has confirmed that a robot's face direction makes a human notice its gaze (Imai et al, 2002) and suggested that its face direction is effective for signaling to whom the robot will talk in a multi-user situation.

As shown in Fig. 9, a tracking function increases robot's mutual gaze chances when subjects may have a feeling that the robot looks at them. In addition, robots with a tracking function influenced decision-making more significantly than robots without it. Thus, such a feeling of being looked at would be necessary for producing some changes on human decision. In other words, our results show that mutual gaze influences not only human feelings but also decision-making in cases of interaction between humans and robots. And, a feeling that a robot looks at us is fundamental and crucial to a robot's becoming a communication partner.

\section{Conclusion}

We experimentally confirmed through quantitative evaluation that the degree of recommendation effect firmly depends on the interaction environment. The results show that a three-dimensional body has some advantage when the interaction environment is a three-dimensional space, but has less advantage in two-dimensional space than a twodimensional body does. This suggests that geometrical consistency between an ESA and the interaction environment plays an important role in communication.

In the 3D world case, we also experimentally confirmed that a tracking function for a robot can play the same important role that gaze has in humans; that is, it can increase the robot's influence on human decision-making through the interaction even if the tracking movement is small.

For a robot as a physical advertisement agent, tracking is an important function. The tracking function successfully makes the mutual gaze ratio greater during interaction; however, the ratio doesn't always have a relationship with the influence on decisionmaking.

Mutual gaze increases the chance when users may have feeling of being looked at, but it is not enough for insuring that a robot gives the feeling. To become a communication partner, a robot needs an effective method for emphasizing the feeling.

\section{References}

Ball, G. \& Breese, J., (1998). Emotion and personality in a conversation character. In: Proceedings of the Workshop on Embodied Conversation Characters.

Becheiraz, P. \& Thalmann, D., (1998). A behavioral animation system for autonomous actors personified by emotions. In: Proceedings of the 1998 Workshop on Embodied Conversational Characters.

Beskow, J. \& McGlashan, S. (1997). Olga - conversational agent with gestures. In: Proceedings of the IJCAI-97 Workshop on Animated Interface Agents: Making them Intelligent.

Bruce, V. \& Young, A. (1998). In the eye of the beholder: The science of face perception: Oxford University Press. 
Cassell, J. \& Thórisson, K. R. (1999). The power of a nod and a glance: Envelope vs. emotional feedback in animated conversational agents. In: Applied Artificial Intelligence. Vol. 13. pp. 519-538.

Imai, M., Kanda, T., Ono, T., Ishiguro H., \& Mase, K. (2002). Robot mediated round table: Analysis of the effect of robot's gaze. In Proceedings of 11th IEEE International Workshop on Robot and Human Communication (RO-MAN2002), 2002, pp. 411-416.

Michael, A. \& Mark, C. (1976) Gaze and Mutual Gaze. Cambridge University Press, UK.

Massaro, D. (1998). Perceiving talking faces: From speech perception to a behavioral principle. MIT Press.

Mizuno, O. \& Nakajima, S. (1998). Synthetic speech/sound control language: Mscl. In: 3rd ESCA/COCOSDA Proceedings of International Workshop on Speech Synthesis. pp. 2126.

Nass, C. \& Gong, L. (1999). Maximized modality or constrained consistency? In: Proceedings of the AVSP' '99 Conference.

Nass, C. \& Isbister, K. (1998). Personality in conversational characters: Building better digital interaction partners using knowledge about human personality preferences and perceptions. In: Proceedings of the 1998 Workshop on Embodied Conversational Characters.

Ono, T., Imai, M. \& Ishiguro, H. (2001). A model of embodied communications with gestures between humans and robots. In: Proceedings of Twenty-third Annual Meeting of the Cognitive Science Society. pp. 732-737.

Reeves, B. \& Nass, C. (1996). The Media Equation. Cambridge University Press.

Shibata, T. \& Tanie, K. (2001). Physical and affective interaction between human and mental commit robot. In: Proceedings of IEEE International Conference on Robotics and Automation. pp. 2572-2577.

Shinozawa, K., Yamato, J., Naya, F. \& Kogure, K. (2001). Quantitative evaluation of effect of embodied conversational agents on user decision. In: Proceedings of HCI International 2001. pp. 998-1002.

Shinozawa, K., Naya, F., \& Kogure, K. \& Yamato, J., Effect of robot's tracking users on human decision making. Proceedings of 2004 IEEE/RSJ International Conference on Intelligent Robots and Systems , pp. 1908-1913, 2004, Sendai, Japan

Shinozawa, K., Naya, F., Yamato, J., \& Kogure, K., Differences in Effect of Robot and Screen Agent Recommendations on Human Decision-Making, IJHCS, Vol. 62/2, pp 267279, 2005.

Sperber, D. \& Wilson, D. (1993). RELEVANCE: Communication and cognition. Oxford: blackwell.

Takeuchi, Y., Katagiri, Y., Nass, C. \& Fogg, B. (1998). Social response and cultural dependency in human-computer interaction. In: Proceedings of PRICAI. pp. 114-123.

Yamato, J., Shinozawa, K., Naya, F.: Effect of Shared-attention on Human-Robot Communication, In Proceedings of ACM/CHI2004 Workshop Shaping Human-Robot Interaction-Understainding the Social Aspects of Intelligent Robotic Products

Yamato, J., Brooks, R., Shinozawa, K., and Naya, F.: Human-Robot Dynamic Social Interaction, NTT Technical Review, Vol.1, No.6, Sep. 2003 


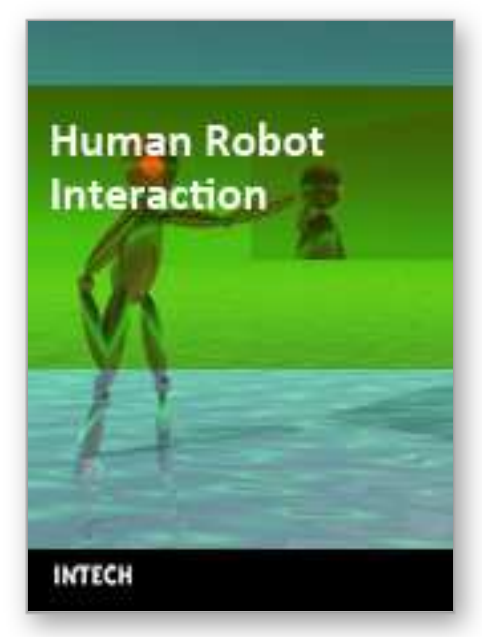

\author{
Human Robot Interaction \\ Edited by Nilanjan Sarkar
}

ISBN 978-3-902613-13-4

Hard cover, 522 pages

Publisher I-Tech Education and Publishing

Published online 01, September, 2007

Published in print edition September, 2007

Human-robot interaction research is diverse and covers a wide range of topics. All aspects of human factors and robotics are within the purview of HRI research so far as they provide insight into how to improve our understanding in developing effective tools, protocols, and systems to enhance HRI. For example, a significant research effort is being devoted to designing human-robot interface that makes it easier for the people to interact with robots. $\mathrm{HRI}$ is an extremely active research field where new and important work is being published at a fast pace. It is neither possible nor is it our intention to cover every important work in this important research field in one volume. However, we believe that $\mathrm{HRI}$ as a research field has matured enough to merit a compilation of the outstanding work in the field in the form of a book. This book, which presents outstanding work from the leading $\mathrm{HRI}$ researchers covering a wide spectrum of topics, is an effort to capture and present some of the important contributions in $\mathrm{HRI}$ in one volume. We hope that this book will benefit both experts and novice and provide a thorough understanding of the exciting field of HRI.

\title{
How to reference
}

In order to correctly reference this scholarly work, feel free to copy and paste the following:

Kazuhiko Shinozawa and Junji Yamato (2007). Effect of Robot and Screen Agent Recommendations on Human Decision-Making, Human Robot Interaction, Nilanjan Sarkar (Ed.), ISBN: 978-3-902613-13-4, InTech, Available from:

http://www.intechopen.com/books/human_robot_interaction/effect_of_robot_and_screen_agent_recommendat ions_on_human_decision-making

\section{INTECH}

open science | open minds

\author{
InTech Europe \\ University Campus STeP Ri \\ Slavka Krautzeka 83/A \\ 51000 Rijeka, Croatia \\ Phone: +385 (51) 770447 \\ Fax: +385 (51) 686166 \\ www.intechopen.com
}

\author{
InTech China \\ Unit 405, Office Block, Hotel Equatorial Shanghai \\ No.65, Yan An Road (West), Shanghai, 200040, China \\ 中国上海市延安西路65号上海国际贵都大饭店办公楼405单元 \\ Phone: +86-21-62489820 \\ Fax: $+86-21-62489821$
}


(C) 2007 The Author(s). Licensee IntechOpen. This chapter is distributed under the terms of the Creative Commons Attribution-NonCommercial-ShareAlike-3.0 License, which permits use, distribution and reproduction for non-commercial purposes, provided the original is properly cited and derivative works building on this content are distributed under the same license. 